\title{
OS FUNDOS DE INVESTIMENTOS EM AÇÕES NO MERCADO FINANCEIRO BRASILEIRO: OS GESTORES E ADMINISTRADORES SÃO CAPAZES DE SUPERAR O ÍNDICE BOVESPA?
}

\section{THE STOCK FUNDS IN BRAZILIAN FINANCIAL MARKET: MANAGERS AND BUSISNESS ADMISTRATORS ARE CAPABLE TO OVERCOME BOVESPA INDEX?}

\author{
Mário Henrique da Fonseca Oliveira* mariohfo@hotmail.com \\ Daisy Aparecida do Nascimento Rebelatto*daisy@usp.sc.br \\ *Universidade de São Paulo - USP
}

\begin{abstract}
Resumo: Neste artigo é realizado um estudo comparativo entre o índice Bovespa e fundos de ações referenciados pelo mesmo, buscando avaliar se os gestores e administradores responsáveis são efetivamente capazes de superar o mercado. As avaliações foram baseadas em conceitos da estatística descritiva, o modelo Capital Asset Price Model, o Trekking Risk e o Value at Risk. Ao todo foram avaliados doze fundos no período de janeiro de 2006 a agosto de 2009. Para cada indicador ou modelo utilizado, foi realizada a comparação individual dos resultados de cada fundo junto ao índice de mercado e junto aos demais fundos, visando identificar as melhores opções para realização de um investimento.
\end{abstract}

Palavras Chave: Fundos de Ações. Índice Bovespa. CAPM. Value at Risk.

Abstract: This paper presents a comparative analysis between Bovespa index and stock funds based into this indicator looking to evaluate if the managers and business administrators, responsible by these funds, are capable to overcome the market. The evaluations are composed by concepts of descriptive statistic, Capital Asset Price Model, Trekking Risk and Value at Risk. Twelve funds were evaluated in the period of January 2006 until August 2009. For each indicator or calc model used, it has been done comparisons between Bovespa index and the others funds, looking for which ones would be better options to invest.

Key-Words: Stock Funds. Bovespa Index. CAPM. Value at Risk.

\section{INTRODUÇÃO}

O mercado de ações mostra-se atraente para investidores que anseiam por lucros maiores que os obtidos nos investimentos em renda fixa, como o CDI e a Poupança. No entanto, o risco inerente aos investimentos em ações, as oscilações, o volume de informações presentes e as habilidades técnicas necessárias para as 
operações neste mercado são fatores que podem inibir parte dos investidores em potencial.

Presente na grande maioria dos bancos de varejo e de investimento, os fundos de ações são geridos por equipes especializadas propondo, em troca de taxas administrativas, uma administração profissionalizada do capital empregado, o que muitas vezes atrai investidores com menor conhecimento no mercado financeiro, ou mesmo, pessoas com tempo restrito para administrar empreendimentos em ações.

Dentre os fundos de investimentos em ações mais comuns encontram-se os fundos que tem como objetivo igualar ou superar o índice de mercado da Bolsa de Valores de São Paulo (lbovespa). Segundo Varga (2001) um gestor capaz de superar o mercado é aquele que tem acesso a melhores informações, e/ou tem modelos para mais bem processar as informações disponíveis para o público em geral.

No entanto, estudos recentes no mercado norte-americano sugerem que os fundos de administração passiva (que seguem a tendência de mercado) têm apresentado melhores resultados quando comparados a fundos de administração ativa (que visam superar o mercado) baseados no índice S\&P500 (WERMERS, 2006 apud CARHART, 1997), trazendo para o cenário nacional uma questão a ser discutida: Os gestores dos fundos de ações brasileiros realmente conseguem atingir seus objetivos, igualando ou superando o retorno proposto pelo índice de mercado?

Buscando ampliar e detalhar esta questão; o presente trabalho tem como objetivo avaliar, frente ao índice Bovespa, a performance dos principais fundos de investimento em ações sob gestão de grandes brancos brasileiros. Tal avaliação utiliza-se de indicadores matemáticos e estatísticos de maneira a comparar junto ao mercado financeiro o retorno sobre o capital investido e o risco envolvido em cada um dos fundos em discussão.

Ao todo doze fundos de investimento em ações foram analisados no período de janeiro de 2006 a agosto de 2009, visando criar um panorama geral do comportamento destes fundos no cenário financeiro nacional. 


\section{OS FUNDOS DE INVESTIMENTOS EM AÇÕES}

Um fundo de investimento é descrito por Assaf Neto (2003) como um conjunto de recursos monetários, formado por depósitos de um grande número de investidores (cotistas), que se destina à aplicação coletiva em carteira de títulos e valores mobiliários. Os fundos de ações mesclam, em suas carteiras, ações e outros ativos. São considerados agressivos, sendo divididos pela Associação Nacional de Bancos de Investimento (ANBID) em seis grupos: Fundos de Ações lbovespa, Fundos de Ações IBrX, Fundos de Ações Setoriais, Fundos de Ações Privatização FGTS - Livre, Fundos de Ações Small Caps, Fundos de Ações Dividendos, Fundos de Ações Sustentabilidade/Governança, Fundos de Ações Livre e Fundos Fechados de Ações.

Este trabalho tem como objetivo avaliar os fundos de Ações Ibovespa, que podem ser subdividos em dois grupos:

- Fundos de Ações Ibovespa Indexado - São fundos cujo objetivo de investimento é acompanhar o comportamento do lbovespa. Não admitem alavancagem, ou seja, não há possibilidade de perda superior ao patrimônio do fundo, desconsiderando-se casos de default nos ativos do fundo (ANBID, 2009).

- Fundos de Ações Ibovespa Ativo - Estes fundos utilizam o Índice Bovespa como referência, tendo o objetivo explícito de superá-lo. Ao contrário dos fundos denominados indexados, este tipo de fundo admite alavancagem (ANBID, 2009).

\section{O ÍNDICE BOVESPA (IBOVESPA)}

O índice da Bolsa de Valores de São Paulo, criado em 1968, é o mais importante indicador de desempenho das cotações das ações negociadas no mercado brasileiro. Seu objetivo básico é refletir o desempenho médio dos negócios à vista, ocorrido nos pregões. Seu cálculo é baseado em uma carteira teórica que procura retratar, da melhor forma possível, o perfil dos negócios realizados na Bovespa. Com o intuito de atualizar a amostra, tornando-a mais representativa, a cada quatro meses a Bolsa de Valores procede a atualização hipotética de sua 
carteira de ações, incluindo os papéis mais representativos do último período e excluindo aqueles de menor participação (ASSAF NETO, 2003).

O cálculo do lbovespa é realizado pelo somatório dos pesos (quantidade teórica da ação multiplicada pelo último preço da mesma) das ações integrantes de sua carteira teórica. Assim sendo, pode ser apurado, a qualquer momento, por meio Expressão 1 (BOVESPA, 2009):

$$
\text { Ibovespa }_{t}=\sum_{i=1}^{n} P_{i, t} \times Q_{i, t}
$$

Em que:

$n$ é o número total de ações componentes da carteira teórica;

$P$ é o ultimo preço da ação " $i$ " no instante $t$;

$Q$ é a quantidade teórica da ação " $i$ " na carteira no instante $t$.

\section{O CONCEITO DE RISCO E RETORNO}

Quando se investe em ações ou em fundos de ações, ao contrário de investimentos de renda fixa, não se sabe ao certo quanto será seu retorno, isto é, se houver retorno. Nada garante que a quantia investida seja recompensada por um bônus esperado. O investimento em ações apresenta risco. Afinal de contas se fosse possível receber sempre exatamente o que se espera, tal investimento seria perfeitamente previsível, portanto, livre de risco.

O risco de um investimento é formado por dois elementos: o risco sistemático ou não diversificável e o risco não sistemático ou diversificável. O risco sistemático é aquele que influencia, em maior ou menor grau, um grande número de ativos. Incertezas sobre condições econômicas gerais, como taxas de juros ou inflação são exemplos de riscos sistemáticos. Já o risco não sistemático, é definido como um risco que afeta, no máximo, um pequeno número de ativos (ROSS et al, 2002).

Segundo conceitos financeiros, o risco não sistemático pode ser eliminado pela diversificação de ativos. Assim, num mercado perfeito só haverá prêmios pelos riscos sistemáticos que o investidor corre.

Neste contexto, para tornar possível a comparação entre os fundos de ações e entre cada um deles e o mercado, fez-se necessária a escolha de indicadores e modelos financeiros e estatísticos que possibilitassem comparar as oscilações e 
riscos inerentes ao mercado. Para contribuir na interpretação dos resultados, os modelos e indicadores foram divididos em três itens: indicadores baseados na estatística descritiva, Capital Asset Price Model, Tracking Risk e Value at Risk.

\section{INDICADORES BASEADOS NA ESTATÍSTICA DESCRITIVA}

A Estatística Descritiva é a parte da Matemática Aplicada que fornece métodos para a análise e interpretação de dados quantitativos para a tomada de decisões junto a amostras ou populações (MONTGOMERY e RUNGER, 2003).

Neste trabalho seus conceitos são utilizados em indicadores para avaliação de desempenho dos fundos de ações no Brasil.

\subsection{Retorno Esperado - $E(R)$}

O Retorno Esperado $E(R)$ é a expectativa futura de um ativo com risco (ROSS et al, 2002). Para efeito de cálculo utiliza-se a média de rentabilidade deste ativo em determinado período, conforme a Expressão 2:

$$
E(R)=\sum_{i=0}^{n} \frac{x_{i}}{N}
$$

Em que:

$x_{i}$ é o retorno do ativo no período $i$;

$N$ é o número de períodos avaliados dos ativos em análise.

\section{$5.2 \operatorname{Risco}(\sigma)$}

O indicador risco quantifica o risco sistemático e o risco não sistemático presente em um ativo. Para se calcular o risco de um investimento deve-se inicialmente calcular sua variância, que é o quadrado da diferença em relação ao retorno esperado. A seguir, multiplica-se cada quadrado de diferenças por sua probabilidade. Soma-se e obtém-se, como resultado, a variância (ROSS et al, 2002). O desvio-padrão ou risco é igual a raiz quadrada da variância.

A variância de uma amostra, denotada por $\sigma^{2}$, de acordo com Montgomery e Runger (2003), é denotada pela Expressão 3: 


$$
\sigma^{2}=\frac{\sum_{i=0}^{N}\left(x_{i}-\mu\right)^{2}}{N}
$$

Em que:

$x_{i}$ é oretorno que o ativo investido no período $i$;

$\mu$ é o retorno esperado do ativo $E(R)$.

\section{3 Coeficiente de Variação (CV)}

O Coeficiente de Variação é uma medida de dispersão relativa usada na comparação do risco de ativos que diferem nos retornos esperados, conforme Expressão 4:

$$
C V=\frac{\sigma}{E(R)}
$$

Quanto mais alto o coeficiente de variação maior será o risco (GITMAN, 2002).

\section{MODELO DE PRECIFICAÇÃO CAPM (CAPITAL ASSET PRICE MODEL)}

A teoria básica que associa o risco e o retorno para todos os ativos é comumente chamada de modelo de formação de preços de ativos de capital (CAPM) (GITMAN, 2002).

Os conceitos envolvidos são apresentados pela Expressão 5:

$$
\left.E\left(R_{i}\right)=E\left(R_{m}\right)+\left[E\left(R_{m}\right)-R_{f}\right)\right] \times \beta_{i}
$$

Em que:

$E\left(R_{i}\right)$ é o retorno exigido pelo ativo;

$E\left(R_{m}\right)$ é retorno de mercado;

$\beta_{i}$ é o índice de risco não diversificável para o ativo $j$;

$R_{f}$ é a taxa de retorno livre de risco.

O coeficiente beta $(\beta)$ é usado para medir o risco não-diversificável. É o índice que mede o grau de movimento do retorno de um ativo em resposta à 
mudança do retorno de mercado. $\mathrm{O} \beta$ de um ativo pode ser encontrado examinandose os retornos históricos do mercado (GITMAN, 2002).

O $\beta$ corresponde à inclinação de uma linha de tendência. Tal linha é obtida através da plotagem dos retornos de mercado versus os retornos do ativo ou grupo de ativos no qual se deseja investir, para um determinado período de tempo.

Matematicamente o $\beta$ pode ser definido pela Expressão 6:

$$
\beta=\frac{\operatorname{cov}\left(R_{n}, R_{m}\right)}{\sigma_{m}^{2}}
$$

Em que:

$\operatorname{cov}\left(R_{n}, R_{m}\right)$ é a covariância do ativo e a carteira de mercado;

$R_{n}$ é o retorno sobre o ativo ou fundo em questão;

$R_{m}$ é o retorno do mercado;

$\sigma_{m}{ }^{2}$ é a variância do retorno sobre a carteira de mercado.

Se um ativo apresenta retorno esperado inferior ao retorno exigido (valor calculado pela Expressão 5) diz-se que ele está supervalorizado, assim sua compra não é recomendada.

\section{TREKING RISK (TR) OU RISCO DE DESCOLAMENTO}

Segundo Oda (2000) o indicador Tracking Risk corresponde ao desvio padrão da série de diferenças entre o retorno que o portfólio propicia e o retorno do benchmark (Ibovespa), conforme apresentado na Expressão 7:

$$
T R=\sigma\left(R_{m}-R_{n}\right)
$$

Em que:

$R_{n}$ é o retorno sobre o ativo ou fundo em questão;

$R_{m}$ é o retorno do mercado;

Quanto maior o seu valor, mais o retorno do fundo se afastou de seu índice de referência. 


\section{VALUE AT RISK (VAR)}

O cálculo do Value at Risk ( $V a R)$ é bastante utilizado como uma forma de gerenciar riscos de mercado pelas instituições financeiras, sendo recomendado pelo Comitê de Basiléia como instrumento para a supervisão bancária (ALMEIDA e GHIRARDI, 1999).

O VaR é a estimativa da quantidade que poderá ser perdida em uma carteira (conjunto de investimentos da instituição financeira), em função das oscilações dos preços dos ativos no mercado, considerado como a pior perda esperada, em um intervalo de tempo, sobre condições normais de mercado em um dado intervalo de confiança (JORION, 1997). A Expressão 8 apresenta seu cálculo para um fundo de investimento baseando-se em dados passados, considerando a perda máxima com 95\% de probabilidade (PASCHOARELI, 2005):

$$
\operatorname{VaR}=I \times[1+E(R)] \times[P(0,05 \leq R)]
$$

Em que:

I é o montante de capital investido;

$E(R)$ é o retorno esperado percentual no período em análise (por exemplo, a média histórica mensal de um determinado ativo);

$[P(0,05 \leq R)]$ é a probabilidade acumulada inversa que representa os $5 \%$ dos piores retornos ocorridos no período em análise (por exemplo, baseando-se em uma distribuição de probabilidade gerada pelos retornos históricos de um ativo; trata-se dos $5 \%$ piores valores de retornos).

A função do VaR é mostrar (com a probabilidade escolhida) qual o menor valor que pode ocorrer no próximo período em caso de prejuízo. Supondo que o montante de capital investido seja $R \$ 10.000,00$; seu VaR pode ser, por exemplo, $R \$ 8.000,00$, o que representa uma perda de capital de $R \$ 2.000,00$.

\section{MÉTODO}

O presente trabalho apresenta uma abordagem quantitativa com objetivo exploratório para avaliação de fundos de investimentos atrelados ao Ibovespa. Os dados financeiros referentes a cada fundo foram coletados em sites de bancos e corretoras nacionais e ajustados para a escala mensal percentual no período de 
janeiro de 2006 a agosto de 2009. Para listagem dos Fundos de Ações em operação no Brasil foi consultado o site da Comissão de Valores Mobiliários (CVM, 2009).

Segundo Gil (1987) quando no objeto de estudo deseja-se confrontar de maneira empírica os dados junto a uma realidade, torna-se necessário traçar um modelo conceitual e operativo para pesquisa, que recebe o nome de delineamento. Há dois grandes tipos de delineamento: o que se vale das chamadas fontes de "papel" e aqueles cujos dados são fornecidos por "pessoas". No primeiro grupo estão a pesquisa bibliográfica e a pesquisa documental. $O$ segundo grupo é compreendido pela pesquisa experimental, a pesquisa ex-post-facto, o levantamento e o estudo de caso.

Entende-se que o delineamento deste trabalho, além do levantamento bibliográfico, também engloba uma pesquisa ex-post-facto, visto que se fazendo uso de informações sobre fatos já concretizados (resultados dos fundos de investimento) para analisá-los e explora-los em detalhes (SILVA e MENEZES, 2001).

A realização dos cálculos de indicadores escolhidos foi feita por meio do software Microsoft $\circledast$ Excel. Também foi necessária a utilização do software estatístico Minitab ${ }^{\circledR} 14.1$ para que fossem efetuados os testes de aderência e cálculos de probabilidade necessários para a aplicação do modelo Value at Risk.

\section{O PROCESSO DE AVALIAÇÃO DOS FUNDOS DE INVESTIMENTOS EM AÇÕES}

Nos próximos sub-itens são apresentados os fundos de investimento avaliados, o período de análise e a aplicação de modelos de indicadores para avaliação.

\subsection{Os Fundos de Investimentos Avaliados e o Período de Análise}

Ao todo foram coletados dados de doze diferentes fundos de investimento em ações, listados no Anexo A. Percebe-se a ausência de fundos de grandes bancos brasileiros (ou de atuação no Brasil), como o Banco do Brasil, o Bradesco, o Citibank, o HSBC e o Santander. Ocorre que em muitos bancos, os dados não são divulgados ou são restritos; por isso, não puderam ser incluídos neste trabalho. 
O período de análise corresponde aos valores percentuais de retorno de cada fundo desde janeiro de 2006 a agosto de 2009. Neste período está presente a crise econômica norte-americana. Embora, em muitos estudos, os períodos de crise sejam eliminados em busca de maior uniformidade para analise, nesta pesquisa acredita-se que a crise deva ser considerada, visto que é inerente à economia mundial e existe a possibilidade de que gestores tenham agido em busca de alguma antecipação ao mercado, reduzindo eventuais perdas.

O Anexo B apresenta os valores ajustados percentualmente, mês a mês, a partir dos dados colhidos nos sites. A evolução mensal do Ibovespa foi obtida por meio da consulta a página http://br.finance.yahoo.com/q/bc?s=\%5EBVSP\&t=5y.

\subsection{A Aplicação da Estatística Descritiva para Avaliação dos Fundos de Ações}

Aplicando os indicadores da estatística descritiva aos dados apresentados no Apêndice B é possível observar o quão dispersos são os retornos dos fundos de investimento em análise e qual deles apresenta maior risco global.

A Tabela 1 apresenta os valores calculados, propondo um ranking aos bancos em função do Coeficiente de Variação apresentado:

Tabela 1 - Avaliação dos Fundos por meio da Estatística Descritiva.

\begin{tabular}{ccccc}
\hline Fundo de Ações & $\boldsymbol{E}(\boldsymbol{R})$ & $\boldsymbol{\sigma}$ & $\boldsymbol{C V}$ & Ranking \\
\hline Real Ativo & $2,275 \%$ & $6,874 \%$ & 3,021 & 1 \\
Real Indexado & $1,514 \%$ & $6,953 \%$ & 4,592 & 2 \\
UBS Pactual Ativo & $1,744 \%$ & $8,224 \%$ & 4,715 & 3 \\
Ibovespa & $1,495 \%$ & $7,657 \%$ & 5,120 & - \\
Votorantim Ativo & $1,520 \%$ & $7,808 \%$ & 5,137 & 4 \\
Banif Ativo & $1,636 \%$ & $9,099 \%$ & 5,560 & 5 \\
Credit Agricole & $1,491 \%$ & $8,311 \%$ & 5,576 & 6 \\
Ativo & $1,309 \%$ & $7,706 \%$ & 5,888 & 7 \\
Itaú Ativo & $1,287 \%$ & $7,728 \%$ & 6,003 & 8 \\
Itaú Indexado & $1,161 \%$ & $7,681 \%$ & 6,616 & 9 \\
Caixa Indexado & $1,086 \%$ & $7,407 \%$ & 6,821 & 10 \\
Banrisul Indexado & $1,081 \%$ & $9,716 \%$ & 8,991 & 11 \\
Credit Suisse Ativo & $0,177 \%$ & $9,653 \%$ & 54,521 & 12 \\
Máxima Ativo & & & & \\
\hline
\end{tabular}

Analisando estes valores, observa-se que apenas três fundos (Real Ativo, Real Indexado e USB Pactual Ativo) apresentaram o coeficiente de variação menor que o Ibovespa, ou seja, apresentam uma relação entre risco e retorno menos 
acentuada do que o indicador de mercado. É perceptível que o fundo denominado Máxima Ativo apresenta um resultado bastante ruim em comparação aos demais fundos, principalmente devido ao fato de seu retorno esperado no período ter sido bastante baixo.

\subsection{A Aplicação do modelo CAPM para avaliação dos Fundos de Ações}

Considerando que os fundos de ações são compostos por vários ativos, a parcela do risco total denominada risco não sistemático, teoricamente, é eliminada pela diversificação. Assim, o risco presente em uma carteira de ações pode ser considerado sistemático (representado pelo coeficiente $\beta$ ), que influencia em menor ou maior grau a todos os ativos que o compõe. Como o $\beta$ de cada fundo indica a correlação dele com o mercado, quanto mais dispersa for a rentabilidade mensal do fundo em relação ao lbovespa maior é seu risco sistemático.

Para aplicação do modelo CAPM faz-se necessário definir a taxa de retorno livre de risco a ser utilizada (Eq.5). Embora a rentabilidade da poupança tenha ganhado destaque na economia nacional, preferiu-se utilizar a taxa de juros do Sistema Especial de Liquidação e de Custódia (SELIC), pois seu valor percentual mensal mostra-se maior (BACEN, 2009; BANCO DO BRASIL, 2009):

Rentabilidade Poupança (Agosto 2009) = 0,55080\% a.m.

Rentabilidade Ajustada Poupança (Agosto 2009) $=[0,55080 \% /(1-15 \%)]$

Rentabilidade Ajustada Poupança (Agosto 2009) = 0,6480\%

Taxa Selic (Agosto 2009) = 0,701\% a.m.

Taxa Selic (Agosto 2009)>Rentabilidade Ajustada Poupança (Agosto 2009) (Eq. 11)

Os rendimentos referentes a investimentos em poupança não são tributáveis, enquanto nos investimentos em títulos SELIC é cobrada uma alíquota de imposto de $15 \%$ sobre o lucro obtido. Desta forma para que ambos os valores fossem comparados em um mesmo patamar, criou-se o indicador Rentabilidade Ajustada Poupança, visto que neste trabalho não é considerada a incidência de impostos.

A Tabela 2 apresenta o ranking dos fundos estudados em função do indicador $E(R)-E\left(R_{i}\right)$, o qual indica o quanto a rentabilidade ultrapassou o retorno exigido pelo fundo, considerando o risco sistemático frente ao mercado. 
Tabela 2 - Avaliação dos Fundos por meio do modelo CAPM.

\begin{tabular}{lccccc}
\hline Fundo de Ações & $\boldsymbol{\beta}$ & $\boldsymbol{E}(\boldsymbol{R})$ & $\boldsymbol{E}\left(\boldsymbol{R}_{\boldsymbol{i}}\right)$ & $\boldsymbol{E}(\boldsymbol{R})-\boldsymbol{E}\left(\boldsymbol{R}_{\boldsymbol{i}}\right)$ & Ranking \\
\hline Real Ativo & 0,78 & $2,275 \%$ & $1,322 \%$ & $0,953 \%$ & 1 \\
UBS Pactual Ativo & 0,80 & $1,744 \%$ & $1,332 \%$ & $0,413 \%$ & 2 \\
Real Indexado & 0,76 & $1,514 \%$ & $1,303 \%$ & $0,211 \%$ & 3 \\
Banif Ativo & 1,12 & $1,636 \%$ & $1,595 \%$ & $0,041 \%$ & 4 \\
Votorantim Ativo & 1,00 & $1,520 \%$ & $1,497 \%$ & $0,023 \%$ & 5 \\
Ibovespa & 1,00 & $1,495 \%$ & $1,495 \%$ & $0,000 \%$ & --- \\
Credit & & & & & \\
Ativo & 1,03 & $1,491 \%$ & $1,521 \%$ & $-0,031 \%$ & 6 \\
Itaú Ativo & 0,99 & $1,309 \%$ & $1,487 \%$ & $-0,178 \%$ & 7 \\
Itaú Indexado & 1,00 & $1,287 \%$ & $1,499 \%$ & $-0,211 \%$ & 8 \\
Caixa Indexado & 1,00 & $1,161 \%$ & $1,492 \%$ & $-0,331 \%$ & 9 \\
Banrisul Indexado & 0,96 & $1,086 \%$ & $1,463 \%$ & $-0,377 \%$ & 10 \\
Credit Suisse Ativo & 1,03 & $1,491 \%$ & $1,521 \%$ & $-0,031 \%$ & 11 \\
Máxima Ativo & 1,09 & $0,177 \%$ & $1,570 \%$ & $-1,393 \%$ & 12 \\
\hline
\end{tabular}

Analisando os valores apresentados na Tabela 2, percebe-se que há alteração de posição no ranking, quando comparados à Tabela 1, sendo que o USB Pactual Ativo assume a posição de segundo melhor fundo. No entanto, a principal diferença é que, agora, cinco dos fundos de ações superaram o Ibovespa, enquanto na análise realizada no item anterior, somente três fundos mostraram-se superiores.

\subsection{A Aplicação do Tracking Risk (TR) para avaliação dos Fundos de Ações}

A aplicação deste indicador permite mensurar a distância que o fundo em análise se desprendeu de seu benchmark. Seu cálculo consiste em mensurar o desvio padrão proveniente dos retornos mensais do lbovespa subtraídos dos retornos mensais do fundo em análise. Assim, fundos mais dispersos em relação ao Ibovespa apresentam o indicador TR maior que os fundos que mantiveram seu comportamento mais próximo. A Tabela 3 apresenta os valores de TR de cada fundo. 
Tabela 3 - Valores de TR de cada fundo.

\begin{tabular}{lcc}
\hline Fundo de Ações & TR & Ranking \\
\hline Real Ativo & $6,8740 \%$ & 1 \\
Real Indexado & $6,9531 \%$ & 2 \\
Banrisul Indexado & $7,4071 \%$ & 3 \\
Caixa Indexado & $7,6811 \%$ & 4 \\
Itaú Ativo & $7,7064 \%$ & 5 \\
Itaú Indexado & $7,7281 \%$ & 6 \\
Votorantim Ativo & $7,8084 \%$ & 7 \\
UBS Pactual Ativo & $8,2242 \%$ & 8 \\
Credit Agricole & $8,3114 \%$ & 9 \\
Ativo & & \\
Banif Ativo & $9,0989 \%$ & 10 \\
Máxima Ativo & $9,6526 \%$ & 11 \\
Credit Suisse Ativo & $9,7160 \%$ & 12 \\
\hline
\end{tabular}

Observa-se que os fundos Real Ativo e Real Indexado ratificam os melhores resultados. Pode-se observar que este indicador não leva em consideração rendimento médio de cada fundo, fazendo com que fundos que foram considerados pouco representativos pela aplicação da estatística descritiva e do modelo CAPM, assumam posição de destaque quando ordenados pelo TR, como o Banrisul Indexado e Caixa Indexado.

\subsection{A Aplicação do Value at Risk (VaR) para avaliação dos Fundos de Ações}

Para aplicação do VaR é necessário descobrir a que tipo de distribuição de probabilidade os retornos no período de cada um dos fundos de ações melhor se encaixam para que seja possível lançar uso dos cálculos de probabilidade. Neste trabalho adotou-se o intervalo de confiança em 95\%, ou seja, $1-0,05$. Para realização do calculo, têm-se os passos utilizados nesta pesquisa:

i. Os valores históricos de cada fundo (Anexo B) foram analisados através da função Stat \Quality Tools \Individual Distribution Identification presente no software Minitab 14.1. Por meio de testes de aderência em quatorze diferentes distribuições de probabilidade, esta função fornece ao usuário qual 
das distribuições é a mais adequada (a de maior $p$-value) e quais são os parâmetros de forma e escala da função proposta;

ii. Obtida as distribuições de probabilidade que representam cada um dos fundos em estudo, faz-se necessário calcular a probabilidade cumulativa inversa referente aos $5 \%$ piores. Por exemplo, todos os valores a esquerda da linha vertical $[P(0,05 \leq R)]$ na Figura 1 correspondem a tais resultados. $\mathrm{O}$ cálculo da probabilidade cumulativa inversa é realizado por meio da função Calc \Probability Distributions do Mintab 14.1;

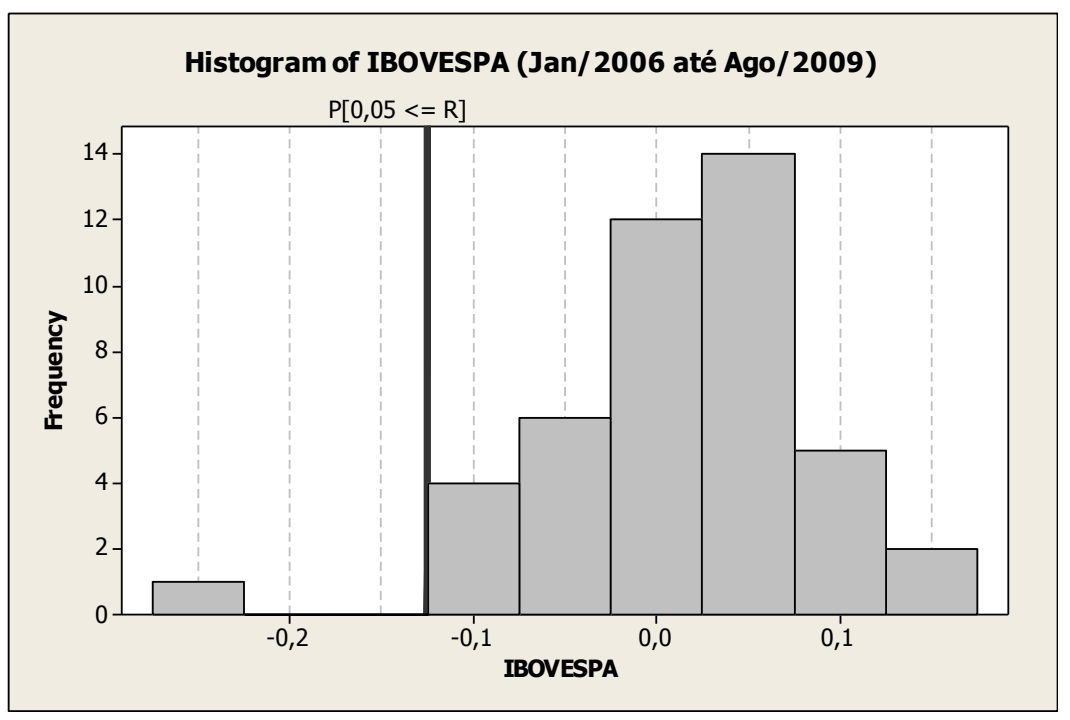

Figura 1 - Histograma dos retornos mensais do lbovespa.

iii. O VaR apresenta qual o montante que representaria a pior perda (neste caso com 95\% confiança) no período seguinte à realização do investimento. Considerando um montante investido $(I)$ de $\mathrm{R} \$ 10.000,00$, o $\mathrm{VaR}$ para cada um dos fundos é obtido aplicando a Eq.8.

A Tabela 4 sintetiza as informações referentes à aplicação do modelo $V a R$, ordenando os fundos em função dos maiores valores em risco. 
Tabela 4 - VaR de cada fundo.

\begin{tabular}{|c|c|c|c|c|c|}
\hline Fundo de Ações & $\begin{array}{c}\text { Dist. de } \\
\text { Probabilidade }\end{array}$ & $p$-value & $\begin{array}{c}P(0,05 \leq \\
R)\end{array}$ & $\operatorname{VaR}$ & Ranking \\
\hline Real Ativo & Logistic & 0,250 & 0,0762326 & $\begin{array}{l}\mathrm{R} \$ \\
9.465,20\end{array}$ & 1 \\
\hline Real Indexado & Logistic & 0,077 & $\overline{0}, 0881104$ & $\begin{array}{l}\mathrm{R} \$ \\
9.270,31\end{array}$ & 2 \\
\hline Ibovespa & Weibull & 0,476 & $-0,124598$ & $\begin{array}{l}R \$ \\
8.903,56\end{array}$ & --- \\
\hline Votorantim Ativo & Weibull & $>0,500$ & $-0,127002$ & $\begin{array}{l}\mathrm{R} \$ \\
8.882,00\end{array}$ & 3 \\
\hline Itaú Ativo & Weibull & $>0,500$ & $-0,126504$ & $\begin{array}{l}\mathrm{R} \$ \\
8.865,85\end{array}$ & 4 \\
\hline Banrisul Indexado & Weibull & $>0,500$ & $-0,124651$ & $\begin{array}{l}\mathrm{R} \$ \\
8.862,08\end{array}$ & 5 \\
\hline UBS Pactual Ativo & Weibull & $>0,500$ & $-0,131686$ & $\begin{array}{l}\mathrm{R} \$ \\
8.857,57\end{array}$ & 6 \\
\hline Itaú Indexado & Weibull & $>0,500$ & $-0,127981$ & $\begin{array}{l}\mathrm{R} \$ \\
8.848,92\end{array}$ & 7 \\
\hline Caixa Indexado & Weibull & $>0,500$ & $-0,128299$ & $\begin{array}{l}\mathrm{R} \$ \\
8.833,11\end{array}$ & 8 \\
\hline Banif Ativo & Weibull & $>0,500$ & $-0,146946$ & $\begin{array}{l}\mathrm{R} \$ \\
8.694,18\end{array}$ & 9 \\
\hline $\begin{array}{l}\text { Credit Agricole } \\
\text { Ativo }\end{array}$ & $\begin{array}{l}\text { Smallest } \\
\text { Value }\end{array}$ & 0,201 & $-0,158318$ & $\begin{array}{l}\mathrm{R} \$ \\
8.565,87\end{array}$ & 10 \\
\hline Credit Suisse Ativo & Weibull & 0,256 & $-0,160848$ & $\begin{array}{l}R \$ \\
8.499,59\end{array}$ & 11 \\
\hline Máxima Ativo & Weibull & $>0,500$ & $-0,172024$ & $\begin{array}{l}\mathrm{R} \$ \\
8.297,46\end{array}$ & 12 \\
\hline
\end{tabular}

Por este critério, ao investir-se a quantia de $\mathrm{R} \$ 10.000,00$ os fundos Real Ativo e Real Indexado apresentariam, dentro da pior perda possível (95\% de confiança), os maiores valores monetários, ou seja; são menos arriscados que os demais fundos, e sobretudo, oferecem um risco menor do que o lbovespa.

Pode-se perceber na coluna $p$-value que todos os valores são maiores do que 0,05; valor mínimo considerado para que a aderência dos dados à distribuição seja significante. Em casos de p-value menores do que 0,05, mantém-se a escolha do maior $p$-value pois, dentre as testadas, trata-se da distribuição de probabilidades que melhor representa os dados, ainda que com menor confiança (MINITAB, 2003).

\section{CONSIDERAÇÕES FINAIS}

Os doze fundos de ações foram avaliados sob a óptica da estatística descritiva, do modelo CAPM, do Tracking Risk e do Value at Risk. Existem diferenças conceituais entre os modelos e indicadores utilizados, porém percebe-se 
que os resultados convergem para uma mesma interpretação: apesar de alguns fundos terem apresentado bons resultados, em âmbito geral, os fundos Real Indexado e Real Ativo destacam-se por efetivamente atingir seus propósitos, superando o desempenho do índice Bovespa, apresentando um menor risco.

Tendo em vista que ambos os fundos são administrados pelo mesmo banco, entende-se que seus gestores utilizam modelos, métodos e políticas mais efetivas para avaliação e execução de investimentos do que os de outras instituições avaliadas na pesquisa.

Embora a atualização quadrimestral do Ibovespa não tenha sido levada em conta neste trabalho, os resultados obtidos sugerem ser mais apropriado investir em uma carteira de ações montada a partir da composição do índice, do que alocar o capital na maior parte dos fundos de ações avaliados, pois além de propiciarem resultados abaixo do esperado, ainda oneram os investimentos por meio de taxas administrativas.

Dentre os indicadores e modelos utilizados neste trabalho, considera-se o VaR e o CAPM os mais representativos conceitualmente. O primeiro considera toda a distribuição de probabilidade obtida a partir do comportamento de cada fundo, o que torna a análise bastante fundamentada. Já o CAPM propicia a correlação do resultado de cada fundo frente ao mercado, conceito que vem ao encontro do objetivo geral do trabalho. A aplicação destes indicadores mostrou divergência nos rankings obtidos, sugerindo que a tomada de decisão, neste tipo de investimento, não deva ser realizada por um modelo único, mas sim por uma avaliação global, como a realizada.

No estudo foram considerados apenas fundos que tomam como referência o índice Bovespa. No entanto, como mostrado, existem outros cinco grupos de investimentos em ações factíveis aos investidores. Para um trabalho futuro, sugerese a avaliação global destes fundos para que seja possível, além de verificar se os gestores efetivamente conseguem cumprir os objetivos administrativos, também avaliar se algum tipo de fundo de investimento em ações apresenta-se mais rentável ou menos arriscado que outros. 


\section{REFERÊNCIAS}

ALMEIDA, A.F. ; GHRARDI, A. Estudo comparativo de modelos de gerenciamento de risco de mercado com uma carteira composta por ativos típicos de um fundo de ações. ENCONTRO NACIONAL DE PÓS-GRADUAÇÃO E PESQUISA EM ADMINISTRAÇÃO, 23. 1999. Anais... 1999.

ANBID. Classificação da ANBID de fundos de investimentos.Disponível em: $<$ www.anbid.com.br/institucional $>$. Acesso em 02 out. 2009.

ASSAF NETO, A. Mercado financeiro. 5. ed. São Paulo: Atlas, 2003.

BANCO DO BRASIL. Rentabilidade de poupança. Disponível em: <http://www21.bb.com.br/portalbb/rendimentosPoupanca/CPR1,2,99.bbx?tipoPesso a=1>. Acesso em: 08 set. 2009.

BACEN. Banco central do Brasil. Disponível em: <http://www.bacen.gov.br>. Acesso em: 02 out. 2009.

BOVESPA. Índice Bovespa definição e metodologia. Disponível em: $<$ http://www.bovespa.com.br/Principal.asp>. Acesso em: 02 out. 2009.

CVM. Comissão de valores mobiliários: consulta a regulamentos e documentos relacionados. Disponível em:

$<$ [http://cvmweb.cvm.gov.br/SWB/Sistemas/SCW/CPublica/ResultBuscaPartic.aspx? TpConsulta=2\&CNPJNome=Ibovespa>. Acesso em: 02 out. 2009.

GIL, A.C. Como elaborar projetos de pesquisa. São Paulo: Atlas, 1987.

GITMAN, L.J. Princípios de administração financeira. 7. ed. São Paulo: Harbra, 2002.

JORION, P. Value at risk: the new benchmark for controlling derivatives risk. 1. ed. California: McGraw-Hill, 1997.

MINITAB® Release 14.1. Minitab Help.

MONTGOMERY D.C. \& RUNGERG, C. Estatística aplicada e probabilidade para engenheiros. 2.ed. Rio de Janeiro: LTC, 2003.

ODA, J.L. análise de persistência de performance dos fundos brasileiros no período de 1995-1998. Dissertação (Mestrado). Faculdade de Economia,

Administração e Contabilidade da Universidade de São Paulo, São Paulo, 2000.

PASCHOARELLI, R. VAR: value at risk: cálculo do var de uma carteira de renda fixa. São Paulo: Saint Paul Institute of Fianance, 2007

ROSS, S.A., WESTERFIELD, R.W., ; JORDAN B. D. Princípios de administração financeira. 2. ed. São Paulo: Atlas, 2002. 
VARGA, G. Índice de sharpe e outros indicadores de performance aplicados a fundos de ações brasileiros. Revista de Administração Contemporânea. Rio de janeiro, n. 3, set./dez., 2001.

SILVA, E.L. ; MENEZES, E.M. Metodologia da pesquisa e elaboração de dissertação. 3. ed. rev. e atual. Florianópolis: LED/UFSC, 2001.

WERMERS, R. Performance evaluation with portfolio holdings information. North American Journal of Economics and Finance, v. 17, p. 207-230, 2006.

YAHOO. Yahoo finanças. Disponível em: <http://www.yahoo.com.br/finance.htm>. Acesso em: 09 out. 2009.

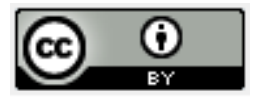

Artigo recebido em 09/11/2009 e aceito para publicação em 09/12/2010. 


\section{Anexo A - Fundos de Investimento em Ações Avaliados}

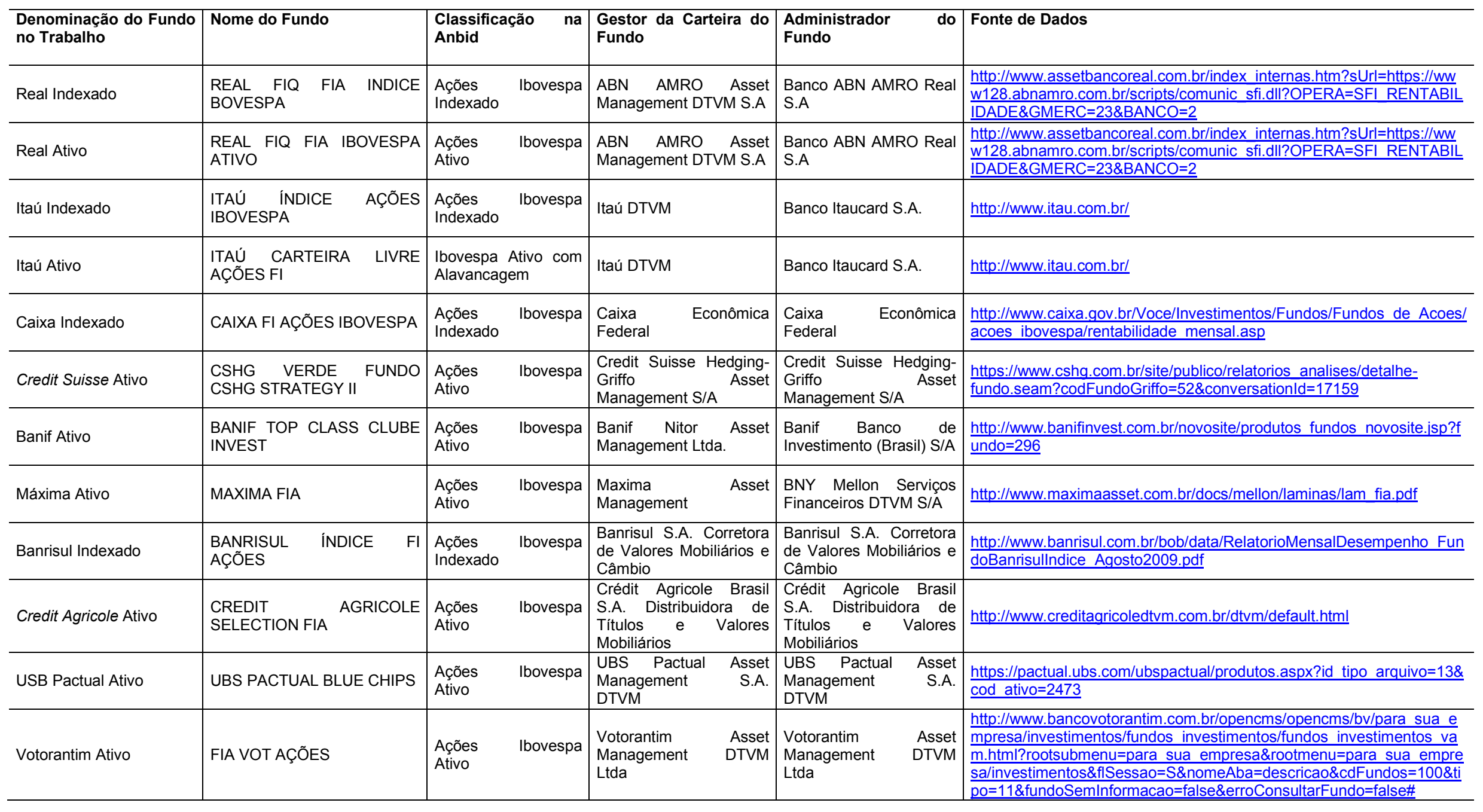


Anexo B - Evolução Mensal Percentual dos Fundos de Investimento em Ações Avaliados

\begin{tabular}{|c|c|c|c|c|c|c|c|c|c|c|c|c|c|}
\hline Data & IBOVESPA & Real Indexado & Real Ativo & Itaú Indexado & Itaú Ativo & Caixa Indexado & Credit Suisse Ativo & Banif Ativo & Máxima Ativo & Banrisul Indexado & Credit Agricole Ativo & UBS Pactual Ativo & Votorantim Ativo \\
\hline $\mathrm{jan} / 06$ & $14,7 \%$ & $13,7 \%$ & $17,9 \%$ & $14,0 \%$ & $16,3 \%$ & $13,9 \%$ & $18,5 \%$ & $8,7 \%$ & $15,3 \%$ & $12,5 \%$ & $16,4 \%$ & $4,0 \%$ & $15,2 \%$ \\
\hline fev/06 & $0,6 \%$ & $1,2 \%$ & $0,9 \%$ & $1,0 \%$ & $0,0 \%$ & $0,9 \%$ & $-0,3 \%$ & $0,2 \%$ & $2,7 \%$ & $0,7 \%$ & $1,8 \%$ & $7,2 \%$ & $2,0 \%$ \\
\hline mar/06 & $-1,7 \%$ & $-1,9 \%$ & $-2,1 \%$ & $-1,8 \%$ & $-1,6 \%$ & $-2,0 \%$ & $-5,3 \%$ & $-0,6 \%$ & $-2,1 \%$ & $-2,5 \%$ & $-3,1 \%$ & $13,4 \%$ & $-2,6 \%$ \\
\hline abr/06 & $6,4 \%$ & $-3,1 \%$ & $6,7 \%$ & $6,2 \%$ & $5,9 \%$ & $5,7 \%$ & $6,9 \%$ & $5,4 \%$ & $5,3 \%$ & $6,3 \%$ & $6,1 \%$ & $-5,4 \%$ & $7,0 \%$ \\
\hline mai/06 & $-9,5 \%$ & $-10,1 \%$ & $-8,9 \%$ & $-9,1 \%$ & $-8,5 \%$ & $-9,2 \%$ & $-12,0 \%$ & $-8,5 \%$ & $-8,4 \%$ & $-9,2 \%$ & $-6,2 \%$ & $6,7 \%$ & $-7,9 \%$ \\
\hline jun/06 & $0,3 \%$ & $1,3 \%$ & $-0,5 \%$ & $-0,2 \%$ & $-0,4 \%$ & $-0,4 \%$ & $-0,2 \%$ & $-4,9 \%$ & $-0,9 \%$ & $-0,4 \%$ & $-0,1 \%$ & $6,1 \%$ & $-0,8 \%$ \\
\hline $\mathrm{jul} / 06$ & $1,2 \%$ & $-2,8 \%$ & $1,2 \%$ & $1,1 \%$ & $0,6 \%$ & $0,9 \%$ & $0,7 \%$ & $0,7 \%$ & $-1,5 \%$ & $0,5 \%$ & $3,6 \%$ & $17,6 \%$ & $0,9 \%$ \\
\hline ago/06 & $-2,3 \%$ & $0,4 \%$ & $-4,7 \%$ & $-2,5 \%$ & $-2,3 \%$ & $-2,6 \%$ & $5,2 \%$ & $1,8 \%$ & $-4,8 \%$ & $-2,1 \%$ & $1,9 \%$ & $0,8 \%$ & $-3,0 \%$ \\
\hline set/06 & $0,6 \%$ & $7,0 \%$ & $0,0 \%$ & $0,1 \%$ & $-1,7 \%$ & $0,1 \%$ & $-0,5 \%$ & $-0,8 \%$ & $-2,2 \%$ & $-0,2 \%$ & $0,9 \%$ & $-1,5 \%$ & $1,3 \%$ \\
\hline out/06 & $7,7 \%$ & $6,3 \%$ & $6,3 \%$ & $6,9 \%$ & $7,0 \%$ & $6,8 \%$ & $7,6 \%$ & $7,4 \%$ & $1,2 \%$ & $6,5 \%$ & $4,2 \%$ & $6,4 \%$ & $6,2 \%$ \\
\hline nov/06 & $6,8 \%$ & $6,3 \%$ & $8,7 \%$ & $6,7 \%$ & $6,2 \%$ & $6,6 \%$ & $5,2 \%$ & $8,6 \%$ & $9,4 \%$ & $6,7 \%$ & $7,7 \%$ & $-8,5 \%$ & $9,3 \%$ \\
\hline dez/06 & $6,1 \%$ & $6,3 \%$ & $7,0 \%$ & $6,1 \%$ & $6,8 \%$ & $6,1 \%$ & $8,2 \%$ & $9,7 \%$ & $4,1 \%$ & $5,5 \%$ & $8,2 \%$ & $-0,5 \%$ & $5,5 \%$ \\
\hline $\mathrm{jan} / 07$ & $0,4 \%$ & $-1,0 \%$ & $1,1 \%$ & $-0,6 \%$ & $0,5 \%$ & $-0,9 \%$ & $1,4 \%$ & $4,2 \%$ & $3,4 \%$ & $-0,4 \%$ & $1,8 \%$ & $0,5 \%$ & $-0,7 \%$ \\
\hline fev/07 & $-1,7 \%$ & $-1,6 \%$ & $0,4 \%$ & $-1,8 \%$ & $-1,9 \%$ & $-1,9 \%$ & $-1,2 \%$ & $0,2 \%$ & $9,1 \%$ & $-1,8 \%$ & $1,4 \%$ & $-1,2 \%$ & $-2,0 \%$ \\
\hline $\mathrm{mar} / 07$ & $4,4 \%$ & $4,1 \%$ & $4,4 \%$ & $4,4 \%$ & $4,6 \%$ & $4,0 \%$ & $1,1 \%$ & $5,6 \%$ & $2,5 \%$ & $4,3 \%$ & $2,8 \%$ & $4,5 \%$ & $3,0 \%$ \\
\hline abr/07 & $6,9 \%$ & $7,2 \%$ & $6,1 \%$ & $7,6 \%$ & $6,6 \%$ & $7,5 \%$ & $8,4 \%$ & $8,9 \%$ & $8,3 \%$ & $7,0 \%$ & $4,2 \%$ & $7,1 \%$ & $7,3 \%$ \\
\hline $\mathrm{mai} / 07$ & $6,8 \%$ & $4,9 \%$ & $6,0 \%$ & $6,3 \%$ & $6,1 \%$ & $6,0 \%$ & $10,9 \%$ & $9,2 \%$ & $18,0 \%$ & $5,9 \%$ & $6,8 \%$ & $6,3 \%$ & $7,5 \%$ \\
\hline jun/07 & $4,1 \%$ & $5,0 \%$ & $4,0 \%$ & $3,6 \%$ & $4,5 \%$ & $3,3 \%$ & $4,9 \%$ & $3,9 \%$ & $1,7 \%$ & $3,3 \%$ & $4,3 \%$ & $4,1 \%$ & $4,4 \%$ \\
\hline jul/07 & $-0,4 \%$ & $0,6 \%$ & $1,3 \%$ & $0,8 \%$ & $2,0 \%$ & $0,7 \%$ & $4,5 \%$ & $3,5 \%$ & $-8,9 \%$ & $1,3 \%$ & $3,8 \%$ & $2,6 \%$ & $3,0 \%$ \\
\hline ago/07 & $0,8 \%$ & $-1,7 \%$ & $-3,8 \%$ & $-1,0 \%$ & $-1,6 \%$ & $-1,3 \%$ & $-3,7 \%$ & $-0,4 \%$ & $-0,1 \%$ & $-0,5 \%$ & $-4,1 \%$ & $-0,5 \%$ & $-3,1 \%$ \\
\hline set/07 & $10,7 \%$ & $10,6 \%$ & $13,2 \%$ & $11,1 \%$ & $9,4 \%$ & $10,6 \%$ & $5,6 \%$ & $13,9 \%$ & $13,9 \%$ & $10,2 \%$ & $10,1 \%$ & $10,6 \%$ & $10,7 \%$ \\
\hline out/07 & $8,0 \%$ & $7,7 \%$ & $8,0 \%$ & $7,8 \%$ & $7,9 \%$ & $6,9 \%$ & $3,8 \%$ & $7,4 \%$ & $6,9 \%$ & $7,2 \%$ & $6,7 \%$ & $7,1 \%$ & $8,0 \%$ \\
\hline nov/07 & $-3,5 \%$ & $-3,0 \%$ & $-2,8 \%$ & $-3,2 \%$ & $-2,5 \%$ & $-2,5 \%$ & $-5,3 \%$ & $-5,5 \%$ & $-10,5 \%$ & $-2,3 \%$ & $-9,5 \%$ & $-3,2 \%$ & $-1,6 \%$ \\
\hline dez/07 & $1,4 \%$ & $0,6 \%$ & $0,8 \%$ & $0,5 \%$ & $0,6 \%$ & $0,6 \%$ & $0,0 \%$ & $1,5 \%$ & $-0,1 \%$ & $1,0 \%$ & $0,8 \%$ & $1,8 \%$ & $3,1 \%$ \\
\hline $\mathrm{jan} / 08$ & $-6,9 \%$ & $-1,0 \%$ & $1,8 \%$ & $-8,4 \%$ & $-8,7 \%$ & $-8,5 \%$ & $-14,2 \%$ & $-11,6 \%$ & $-19,1 \%$ & $-8,0 \%$ & $-13,8 \%$ & $-8,2 \%$ & $-7,6 \%$ \\
\hline fev/08 & $6,7 \%$ & $-1,6 \%$ & $-0,3 \%$ & $8,7 \%$ & $9,4 \%$ & $8,8 \%$ & $10,3 \%$ & $14,5 \%$ & $8,9 \%$ & $8,3 \%$ & $7,4 \%$ & $8,9 \%$ & $8,9 \%$ \\
\hline $\mathrm{mar} / 08$ & $-4,0 \%$ & $4,1 \%$ & $4,4 \%$ & $-5,3 \%$ & $-5,8 \%$ & $-5,7 \%$ & $-8,5 \%$ & $-3,6 \%$ & $-10,0 \%$ & $-5,5 \%$ & $-8,2 \%$ & $-5,6 \%$ & $-6,2 \%$ \\
\hline $\mathrm{abr} / 08$ & $11,3 \%$ & $7.2 \%$ & $6.1 \%$ & $9,0 \%$ & $8.2 \%$ & $8.3 \%$ & $8,9 \%$ & $10,8 \%$ & $6,4 \%$ & $10,1 \%$ & $8,6 \%$ & $9.7 \%$ & $10,4 \%$ \\
\hline mai/08 & $7,0 \%$ & $6,4 \%$ & $6,0 \%$ & $9,2 \%$ & $10,3 \%$ & $9,2 \%$ & $17,9 \%$ & $12,6 \%$ & $13,2 \%$ & $9,9 \%$ & $10,0 \%$ & $9,8 \%$ & $8,6 \%$ \\
\hline jun/08 & $-10,4 \%$ & $3,4 \%$ & $4,0 \%$ & $-10,6 \%$ & $-9,0 \%$ & $-10,7 \%$ & $-11,3 \%$ & $-10,1 \%$ & $-11,2 \%$ & $-9,9 \%$ & $-10,6 \%$ & $-10,9 \%$ & $-10,8 \%$ \\
\hline $\mathrm{jul} / 08$ & $-8,5 \%$ & $0,6 \%$ & $1,3 \%$ & $-8,7 \%$ & $-11,3 \%$ & $-8,8 \%$ & $-9,1 \%$ & $-13,3 \%$ & $-11,7 \%$ & $-8,6 \%$ & $-9,8 \%$ & $-9,9 \%$ & $-9,4 \%$ \\
\hline ago/08 & $-6,4 \%$ & $-1,7 \%$ & $-1,3 \%$ & $-6,7 \%$ & $-6,8 \%$ & $-6,9 \%$ & $-14,1 \%$ & $-7,0 \%$ & $-8,2 \%$ & $-6,2 \%$ & $-8,1 \%$ & $-7,6 \%$ & $-7,0 \%$ \\
\hline set/08 & $-11,0 \%$ & $-11,5 \%$ & $-10,7 \%$ & $-11,3 \%$ & $-11,0 \%$ & $-11,5 \%$ & $-20,4 \%$ & $-16,1 \%$ & $-19,0 \%$ & $-9,6 \%$ & $-11,2 \%$ & $-11,8 \%$ & $-9,3 \%$ \\
\hline out/08 & $-24,8 \%$ & $-24,8 \%$ & $-23,8 \%$ & $-25,0 \%$ & $-24,1 \%$ & $-25,0 \%$ & $-26,2 \%$ & $-30,1 \%$ & $-28,8 \%$ & $-24,9 \%$ & $-25,1 \%$ & $-25,5 \%$ & $-25,0 \%$ \\
\hline nov/08 & $-1,8 \%$ & $-2,0 \%$ & $-0,2 \%$ & $-1,9 \%$ & $-0,5 \%$ & $-1,7 \%$ & $2,5 \%$ & $-1,9 \%$ & $-2,7 \%$ & $-3,0 \%$ & $0,3 \%$ & $-2,8 \%$ & $-4,5 \%$ \\
\hline dez/08 & $2,6 \%$ & $-2,5 \%$ & $1,6 \%$ & $2,3 \%$ & $1,5 \%$ & $2,4 \%$ & $-0,8 \%$ & $4,3 \%$ & $-4,2 \%$ & $1,5 \%$ & $5,3 \%$ & $2,8 \%$ & $4,1 \%$ \\
\hline jan/09 & $4,7 \%$ & $-4,3 \%$ & $4,1 \%$ & $4,4 \%$ & $2,7 \%$ & $4,3 \%$ & $2,4 \%$ & $11,1 \%$ & $1,0 \%$ & $3,0 \%$ & $3,8 \%$ & $4,4 \%$ & $3,6 \%$ \\
\hline fev/09 & $-2,8 \%$ & $-3,1 \%$ & $-2,4 \%$ & $-3,1 \%$ & $-2,0 \%$ & $-3,1 \%$ & $-2,3 \%$ & $-2,9 \%$ & $-1,2 \%$ & $-2,8 \%$ & $0,5 \%$ & $-2,9 \%$ & $-0,8 \%$ \\
\hline $\mathrm{mar} / 09$ & $7,2 \%$ & $6,8 \%$ & $6,7 \%$ & $6,9 \%$ & $7,8 \%$ & $6,9 \%$ & $3,0 \%$ & $7,1 \%$ & $0,5 \%$ & $5,9 \%$ & $8,1 \%$ & $6,6 \%$ & $7,7 \%$ \\
\hline $\mathrm{abr} / 09$ & $15,6 \%$ & $15,2 \%$ & $14,0 \%$ & $15,3 \%$ & $14,8 \%$ & $15,3 \%$ & $21,8 \%$ & $14,2 \%$ & $6,4 \%$ & $14,4 \%$ & $18,6 \%$ & $15,3 \%$ & $15,5 \%$ \\
\hline $\mathrm{mai} / 09$ & $12,5 \%$ & $12,1 \%$ & $11,7 \%$ & $12,2 \%$ & $11,0 \%$ & $12,3 \%$ & $13,2 \%$ & $10,7 \%$ & $7,7 \%$ & $11,1 \%$ & $11,5 \%$ & $11,9 \%$ & $11,1 \%$ \\
\hline jun/09 & $-3,3 \%$ & $-3,5 \%$ & $-2,5 \%$ & $-3,4 \%$ & $-4,0 \%$ & $-3,5 \%$ & $-1,1 \%$ & $-5,9 \%$ & $-1,7 \%$ & $-4,2 \%$ & $-3,1 \%$ & $-2,6 \%$ & $-2,9 \%$ \\
\hline $\mathrm{jul} / 09$ & $6,4 \%$ & $6,1 \%$ & $6,4 \%$ & $6,2 \%$ & $7,5 \%$ & $6,0 \%$ & $8,7 \%$ & $6,6 \%$ & $11,6 \%$ & $4,8 \%$ & $8,1 \%$ & $6,1 \%$ & $6,0 \%$ \\
\hline ago/09 & $3,1 \%$ & $2,8 \%$ & $1,9 \%$ & $3,0 \%$ & $3,3 \%$ & $2,8 \%$ & $2,5 \%$ & $2,5 \%$ & $7,5 \%$ & $2,1 \%$ & $3,0 \%$ & $2,9 \%$ & $2,1 \%$ \\
\hline
\end{tabular}

